\title{
On the very nature of neutrinos: the $\beta$-decay as a test bench
}

\section{G. G. Luciano*}

Università di Salerno, Via Giovanni Paolo II, 132 I-84084 Fisciano (SA), Italy.

INFN, Sezione di Napoli, Gruppo collegato di Salerno, Italy.

E-mail: glucianodsa.infn.it

\begin{abstract}
We discuss the rôle of neutrino mixing in the context of the neutron $\beta$-decay. Calculations are performed for both static and accelerated neutrons. The lepton charge conservation in the neutrino production/detection vertices, which is expected in the tree level approximation of the Standard Model, is taken as our starting point. On this basis, we manage to show that the correct way to describe the emitted (anti-)neutrino is by use of flavor (rather than mass) eigenstates, confirming previous results in the literature.
\end{abstract}

Corfu Summer Institute 2019 "School and Workshops on Elementary Particle Physics and Gravity" (CORFU2019)

31 August - 25 September 2019

Corfu, Greece

${ }^{*}$ Speaker. 


\section{Introduction}

In a series of recent works $[1,2,3,4,5,6,7]$, the inverse $\beta$-decay of uniformly accelerated protons, $p \rightarrow n+e^{+}+v_{e}$, has served as a theoretical tool for testing the very nature of asymptotic neutrino states - mass or flavor. By computing the scalar decay rate both in the laboratory frame (where the proton is accelerated) and in the comoving frame (where the proton is at rest and interacts with the Unruh [8] thermal bath of electrons and antineutrinos), it has been shown that the only way to get consistency with: $i$ ) the general covariance of Quantum Field Theory (QFT) [2], ii) the phenomenon of neutrino oscillations [6] and iii) the related effects of CP violation [7] is by use of flavor states. However, due to the lack of feasible experiments ${ }^{1}$ and the widespread skepticism on the realness of Unruh effect, one may question the physical relevance of the above framework and the results it entails [4].

To avoid this kind of ambiguity, here we consider a more experimentally accessible process, i.e. the neutron $\beta$-decay

$$
n \rightarrow p+e^{-}+\bar{v}_{e}
$$

Unlike protons, it is well-known that isolated neutrons decay spontaneously (i.e. no acceleration is needed) with a mean lifetime of about $887 \mathrm{~s}$, according to the Standard Model. Hence, the advantage of this study is that one can strip the formalism down to essentials at first, getting rid of any extra ingredient which may overshadow the real core of the analysis (we shall refer to this streamlined analysis as minimal analysis). Calculations can then be refined step-by-step, bringing into play new (higher-order) effects, such as the acceleration of the neutron or the ensuing thermal Unruh effect. Clearly, this bottom-up approach makes no sense for the proton: in that case, indeed, the stability of the particle trivializes the minimal analysis.

Along this line, in what follows we start by investigating the rôle of neutrino mixing in the $\beta$-decay of static neutrons. Calculations of the transition rate and energy spectrum are performed within Pontecorvo theory with only two neutrino flavors [9]. To guarantee the family lepton number conservation in the interaction vertices, we take the emitted antineutrino to be in a flavor eigenstate, showing that our results are in agreement with the predictions of the minimal Standard Model with massless neutrino in the relativistic limit. We also comment on the possibility to naturally account for flavor oscillations within such a framework. The above analysis is later generalized to accelerated neutrons by using the semiclassical treatment of Refs. $[2,6,7] .^{2}$ Apart from its intrinsic interest in some astrophysical situations as, e.g., the cooling of neutron stars, where significant accelerations can be provided by internal magnetic fields, we remark that this study is intended as a "stress-test" of the formalism of Refs. [2, 6, 7]. In the limit of vanishing acceleration, indeed, we prove that the obtained decay rate recovers the standard expression for static neutrons, thus providing a check for the internal consistency of the formalism of Refs. [2, 6, 7], as well as for the results there contained. We finally discuss the relevance of our outcome to some recent neutrino experiments such as KATRIN and PTOLEMY.

\footnotetext{
${ }^{1}$ We recall that protons are not likely to decay in laboratory conditions, e.g. with typical accelerations at LHC/CERN, a rough estimation of their lifetime yields $\tau_{p} \approx 10^{3 \times 10^{8}} \mathrm{yr}$, a time out of reach even for the most longlived physicist.

${ }^{2}$ A similar analysis with massless neutrino was proposed in Ref. [10].
} 
The remainder of the work is organized as follows: in Sec. 2 we review the basics of Pontecorvo theory of neutrino mixing and set the stage for the analysis of the $\beta$-decay. Section 3 is devoted to compute the decay rate of both static and accelerated neutrons. Conclusions and future prospects are summarized in Sec. 4 . Throughout all the work, we shall use natural units $\hbar=c=1$ and the mostly negative metric signature

$$
\eta^{\mu v}=(1,-1,-1,-1)
$$

\section{Neutron $\beta$-decay and neutrino mixing: the general framework}

In the Standard Model of fundamental interactions, neutrinos weakly couple with other particles in flavor eigenstates $\left|v_{\ell}\right\rangle(\ell=e, \mu)$, which are linear superpositions of mass eigenstates $\left|v_{J}\right\rangle$ $(j=1,2)$ via Pontecorvo mixing transformation [9]

$$
\left(\begin{array}{l}
\left|v_{e}\right\rangle \\
\left|v_{\mu}\right\rangle
\end{array}\right)=\left(\begin{array}{cc}
\cos \theta & \sin \theta \\
-\sin \theta & \cos \theta
\end{array}\right)\left(\begin{array}{l}
\left|v_{1}\right\rangle \\
\left|v_{2}\right\rangle
\end{array}\right)
$$

with $\theta$ being the mixing angle (a similar transformation holds for neutrino fields as well ${ }^{3}$ ). The fact that the mass and flavor representations cannot be simultaneously diagonalized begs the question of which one should be regarded as fundamental when computing observables such as cross-sections or decay rates within the $S$-matrix framework. In spite of this ambiguity, a clue to the solution was recently given in Ref. [12], where it was shown that the use of flavor neutrinos as external states is well-founded both physically (since it leads to predictions which are in agreement with the Standard Model) and mathematically (as the asymptotic $t \rightarrow \pm \infty$ limit does not pose any technical difficulties in performing the computations). Additionally, this setting allows to naturally incorporate the effects of flavor oscillations, a feature the treatment with mass states would fail to pinpoint [6]. Thus, based on the above considerations, in what follows we shall resort to the flavor representation for developing our calculations.

In order to describe the $\beta$-decay (1.1), let us employ a Fermi-like effective theory with currents defined in a covariant manner [10]. For this purpose, we consider a neutron in Minkowski spacetime of inertial coordinates $x \equiv(t, \mathbf{x}) \in \mathbb{R}^{4}$. Denoting by $x^{\alpha}(\tau)$ the neutron worldline of proper time $\tau$, the associated current takes the form

$$
\hat{j}_{n}^{\alpha}(x)=\frac{\hat{q}(\tau) u^{\alpha}(\tau)}{u^{0}(\tau)} \delta^{3}[\mathbf{x}-\mathbf{x}(\tau)]
$$

where $u^{0}=d t / d \tau$ and $\hat{q}(\tau)=e^{i \hat{H}_{0} \tau} \hat{q}_{0} e^{-i \hat{H}_{0} \tau}$ is the monopole operator, with $\hat{H}_{0}$ being the neutron proper Hamiltonian [10].

The current $\hat{j}^{\alpha}$ in Eq. (2.2) depends in an essential way on the motion of the neutron. In principle, a similar expression should be defined for the proton as well. In what follows, however,

\footnotetext{
${ }^{3}$ In Ref. [11] it was shown that the implementation of Pontecorvo transformations at level of fields leads to an inconsistency with the definition (2.1) of flavor states, which actually turn out to be generalized coherent states. However, in the relativistic regime and for small mass-difference between mixed neutrinos, Eq. (2.1) well approximate the exact field theoretical definition (see, for instance, Refs. [3, 5]).
} 
we shall work in the so-called "no-recoil approximation", namely we assume the four-velocity of the proton to be insensitive to the electron-antineutrino emission. This holds true as far as the momenta of the emitted leptons are much smaller than the masses of neutron and proton. Within such a framework, we can naively neglect the different inner structures of the two hadrons and regard them as energy levels of a unique quantum system, the nucleon, moving along a fixed trajectory. The neutron current $\hat{j}_{n}^{\alpha}$ is then identified with the semiclassical nucleon current $\hat{j}_{n \rightarrow p}^{\alpha}$, the matrix element of which reads

$$
\begin{aligned}
j_{n \rightarrow p}^{\alpha}(x) & \equiv\left\langle p\left|\hat{j}_{n \rightarrow p}^{\alpha}(x)\right| n\right\rangle \\
& =G_{e f f} e^{-i\left(M_{n}-M_{p}\right) \tau} \frac{u^{\alpha}(\tau)}{u^{0}(\tau)} \delta^{3}[\mathbf{x}-\mathbf{x}(\tau)],
\end{aligned}
$$

where $G_{\text {eff }}=\left|\left\langle p\left|\hat{q}_{0}\right| n\right\rangle\right|$ is the effective coupling constant and $|n\rangle(|p\rangle)$ is the neutron (proton) energy eigenstate of eigenvalue $M_{n(p)}$, i.e. $\hat{H}_{0}|n\rangle(|p\rangle)=M_{n(p)}|n\rangle(|p\rangle)$.

On the other hand, we adopt a full quantum picture to describe the emitted electron and antineutrino by defining the lepton current as

$$
\hat{j}_{l}^{\alpha}(x)=\sum_{\ell=e, \mu}\left(\hat{\Psi}_{v_{\ell}}(x) \gamma^{\alpha}\left(1-\gamma^{5}\right) \hat{\Psi}_{\ell}(x)+\hat{\bar{\Psi}}_{\ell}(x) \gamma^{\alpha}\left(1-\gamma^{5}\right) \hat{\Psi}_{v_{\ell}}(x)\right)
$$

where $\gamma^{\alpha}$ are the Dirac matrices in the Dirac representation [2]. ${ }^{4}$ The neutrino and electron field, $\hat{\Psi}_{v_{\ell}}$ and $\hat{\Psi}_{\ell}$, respectively, are quantized according to

$$
\hat{\Psi}(x)=\sum_{\sigma= \pm} \int d^{3} \mathbf{k}\left[\hat{b}_{\mathbf{k} \sigma} \psi_{\mathbf{k} \sigma}^{(+\omega)}(x)+\hat{d}_{\mathbf{k} \sigma}^{\dagger} \psi_{-\mathbf{k}-\sigma}^{(-\omega)}(x)\right]
$$

where

$$
\psi_{\mathbf{k} \sigma}^{( \pm \omega)}(x)=\frac{e^{i(\mp \omega t+\mathbf{k} \cdot \mathbf{x})}}{2^{2} \pi^{\frac{3}{2}}} u_{\sigma}^{( \pm \omega)}(\mathbf{k})
$$

The spinors $u_{\sigma}^{( \pm \omega)}(\mathbf{k})$ are given by

$$
u_{+}^{( \pm \omega)}(\mathbf{k})=\frac{1}{\sqrt{\omega(\omega \pm m)}}\left(\begin{array}{c}
m \pm \omega \\
0 \\
k^{z} \\
k^{x}+i k^{y}
\end{array}\right), \quad u_{-}^{( \pm \omega)}(\mathbf{k})=\frac{1}{\sqrt{\omega(\omega \pm m)}}\left(\begin{array}{c}
0 \\
m \pm \omega \\
k^{x}-i k^{y} \\
-k^{z}
\end{array}\right)
$$

The operators $\hat{b}_{\mathbf{k} \sigma}\left(\hat{d}_{\mathbf{k} \sigma}\right)$ in Eq. (2.5) denote the annihilators of particles (antiparticles) having threemomentum $\mathbf{k} \equiv\left(k^{x}, k^{y}, k^{z}\right)$, polarization $\sigma= \pm$, frequency $\omega=\sqrt{\mathbf{k}^{2}+m^{2}}$ and mass $m$. Since the modes (2.6) are normalized to Dirac delta [2], these operators obey the canonical anticommutation relations.

\footnotetext{
${ }^{4}$ In the case of a static neutron, it is well-known that a purely quantum definition in terms of the up and down quark fields can be adopted for the hadronic current as well. Hence, the use of the semiclassical expression (2.2) in the present analysis arises out of the attempt of formulating a description of the $\beta$-decay which can be applied to static, as well as accelerated neutrons (for which a full-fledged field theoretical treatment that also accounts for the external accelerating agent is still missing).
} 
To compute the neutron $\beta$-decay spectrum at tree level, we now couple the nucleon and lepton currents through the weak-interaction action

$$
\hat{S}_{I}=\int d^{4} x \sqrt{-g(x)} \hat{j}_{n \rightarrow p, \alpha}(x) \hat{j}_{l}^{\alpha}(x)
$$

with $g$ being the determinant of the metric. Accordingly, the vacuum transition amplitude can be evaluated as

$$
\mathscr{A}^{n \rightarrow p}\left(\bar{v}_{e}\right)=\left\langle p\left|\otimes\left\langle e^{-}, \bar{v}_{e}\left|\hat{S}_{I}\right| 0\right\rangle \otimes\right| n\right\rangle,
$$

where $\bar{v}_{e}$ on the 1.h.s. reminds us that we are analyzing the process with an electron antineutrino among the detected particles.

Now, by plugging Eq. (2.8) into (2.9) with the field decomposition (2.5), we obtain

$$
\begin{aligned}
\mathscr{A}^{n \rightarrow p}\left(\bar{v}_{e}\right)= & \cos ^{2} \theta \int d^{4} x \sqrt{-g} j_{n \rightarrow p, \alpha} \bar{\psi}_{\mathbf{k}_{e}, \sigma_{e}}^{\left(+\omega_{e}\right)} \gamma^{\alpha}\left(1-\gamma^{5}\right) \psi_{-\mathbf{k}_{v},-\sigma_{v}}^{\left(-\omega_{v_{v}}\right)} \\
& +\sin ^{2} \theta \int d^{4} x \sqrt{-g} j_{n \rightarrow p, \alpha} \bar{\psi}_{\mathbf{k}_{e}, \sigma_{e}}^{\left(+\omega_{e}\right)} \gamma^{\alpha}\left(1-\gamma^{5}\right) \psi_{-\mathbf{k}_{v},-\sigma_{v}}^{\left(-\omega_{v_{2}}\right)}
\end{aligned}
$$

where the spacetime dependence on the r.h.s. has been omitted in order to streamline the notation. Note that, since the interaction is the standard electroweak vertex producing the antineutrino with definite flavor, we have resorted to the transformation (2.1) to express both the antineutrino field and state in terms of the corresponding massive objects. Moreover, we have employed the equalmomentum and equal-polarization assumptions for states with different masses.

Equation (2.10) allows us to evaluate the averaged differential transition probability as

$$
\frac{d \mathscr{P}^{n \rightarrow p}}{d^{3} \mathbf{k}_{v} d^{3} \mathbf{k}_{e}}\left(\bar{v}_{e}\right)=\frac{1}{2} \sum_{\sigma_{e}, \sigma_{v}= \pm}\left|\mathscr{A}^{n \rightarrow p}\right|^{2} .
$$

By explicit calculations, one can show that

$$
\begin{aligned}
& \frac{d \mathscr{P}^{n \rightarrow p}}{d^{3} \mathbf{k}_{v} d^{3} \mathbf{k}_{e}}\left(\bar{v}_{e}\right)=\frac{1}{2} \cos ^{4} \theta \int d^{4} x \sqrt{-g(x)} \int d^{4} x^{\prime} \sqrt{-g\left(x^{\prime}\right)} J_{\alpha \beta}^{n \rightarrow p}\left(x, x^{\prime}\right) G_{\mathbf{k}_{e}, \mathbf{k}_{v}}^{\alpha \beta(1)}\left(x, x^{\prime}\right) \\
& +\frac{1}{2} \sin ^{4} \theta \int d^{4} x \sqrt{-g(x)} \int d^{4} x^{\prime} \sqrt{-g\left(x^{\prime}\right)} J_{\alpha \beta}^{n \rightarrow p}\left(x, x^{\prime}\right) G_{\mathbf{k}_{e}, \mathbf{k}_{v}}^{\alpha \beta(2)}\left(x, x^{\prime}\right) \\
& +\frac{1}{2} \cos ^{2} \theta \sin ^{2} \theta\left(\int d^{4} x \sqrt{-g(x)} \int d^{4} x^{\prime} \sqrt{-g\left(x^{\prime}\right)} J_{\alpha \beta}^{n \rightarrow p}\left(x, x^{\prime}\right) G_{\mathbf{k}_{e}, \mathbf{k}_{v}}^{\alpha \beta(1,2)}\left(x, x^{\prime}\right)+\text { c.c. }\right),
\end{aligned}
$$

where

$$
J_{\alpha \beta}^{n \rightarrow p}\left(x, x^{\prime}\right)=G_{e f f}^{2} \frac{u_{\alpha}(\tau) u_{\beta}\left(\tau^{\prime}\right)}{u^{0}(\tau) u^{0}\left(\tau^{\prime}\right)} e^{-i \Delta M\left(\tau-\tau^{\prime}\right)} \delta^{3}[\mathbf{x}-\mathbf{x}(\tau)] \delta^{3}\left[\mathbf{x}^{\prime}-\mathbf{x}\left(\tau^{\prime}\right)\right],
$$

with $\Delta M=M_{n}-M_{p} \simeq 1.29 \mathrm{MeV}$, and

$$
\begin{aligned}
G_{\mathbf{k}_{e}, \mathbf{k}_{v}}^{\alpha \beta(j)}\left(x, x^{\prime}\right) & =\sum_{\sigma_{e}, \sigma_{v}= \pm}\left[\bar{\psi}_{\mathbf{k}_{e}, \sigma_{e}}^{\left(+\omega_{e}\right)}(x) \gamma^{\alpha}\left(1-\gamma^{5}\right) \psi_{-\mathbf{k}_{v},-\sigma_{v}}^{\left(-\omega_{v}\right)}(x) \bar{\psi}_{-\mathbf{k}_{v},-\sigma_{v}}^{\left(-\omega_{v_{j}}\right)}\left(x^{\prime}\right) \gamma^{\beta}\left(1-\gamma^{5}\right) \psi_{\mathbf{k}_{e}, \sigma_{e}}^{\left(+\omega_{e}\right)}\left(x^{\prime}\right)\right], \\
G_{\mathbf{k}_{e}, \mathbf{k}_{v}}^{\alpha \beta(i, j)}\left(x, x^{\prime}\right) & =\sum_{\sigma_{e}, \sigma_{v}= \pm}\left[\bar{\psi}_{\mathbf{k}_{e}, \sigma_{e}}^{\left(+\omega_{e}\right)}(x) \gamma^{\alpha}\left(1-\gamma^{5}\right) \psi_{-\mathbf{k}_{v},-\sigma_{v}}^{\left(-\omega_{v_{v}}\right)}(x) \bar{\psi}_{-\mathbf{k}_{v},-\sigma_{v}}^{\left(-\omega_{v_{j}}\right)}\left(x^{\prime}\right) \gamma^{\beta}\left(1-\gamma^{5}\right) \psi_{\mathbf{k}_{e}, \sigma_{e}}^{\left(+\omega_{e}\right)}\left(x^{\prime}\right)\right],
\end{aligned}
$$


with $(i, j)=\{1,2\}$. The first of these two equations can be cast in the form

$$
\begin{aligned}
G_{\mathbf{k}_{e}, \mathbf{k}_{v}}^{\alpha \beta(j)}\left(x, x^{\prime}\right)= & \operatorname{tr}\left\{\gamma^{\alpha}\left(1-\gamma^{5}\right) \sum_{\sigma_{v}= \pm}\left[\psi_{-\mathbf{k}_{v},-\sigma_{v}}^{\left(-\omega_{v_{j}}\right)}(x) \bar{\psi}_{-\mathbf{k}_{v},-\sigma_{v}}^{\left(-\omega_{v_{j}}\right)}\left(x^{\prime}\right)\right]\right. \\
& \left.\times \gamma^{\beta}\left(1-\gamma^{5}\right) \sum_{\sigma_{e}= \pm}\left[\psi_{\mathbf{k}_{e}, \sigma_{e}}^{\left(\omega_{e}\right)}\left(x^{\prime}\right) \bar{\psi}_{\mathbf{k}_{e}, \sigma_{e}}^{\left(\omega_{e}\right)}(x)\right]\right\},
\end{aligned}
$$

which can be further simplified by using the spin-sum relation

$$
\sum_{\sigma= \pm} \psi_{ \pm \mathbf{k}, \sigma}^{( \pm \omega)}(x) \bar{\psi}_{ \pm \mathbf{k}, \sigma}^{( \pm \omega)}\left(x^{\prime}\right)=\frac{(k \pm m)}{2(2 \pi)^{3} \omega} e^{ \pm i k^{\lambda}\left(x-x^{\prime}\right)}
$$

where $k^{\lambda} \equiv(\omega, \mathbf{k})$ is the four-momentum of the emitted fermion and $k k^{\lambda} \gamma_{\lambda}$ in the usual Feynman slash notation. On the other hand, $G_{\mathbf{k}_{e}, \mathbf{k}_{v}}^{\alpha \beta(i, j)}$ is less easy to handle, as it involves the product of neutrino spinors with different masses. We shall face it explicitly on a case-by-case basis.

Substitution of Eqs. (2.13) - (2.17) into (2.12) yields

$$
\begin{aligned}
& \frac{d \mathscr{P}^{n \rightarrow p}}{d^{3} \mathbf{k}_{v} d^{3} \mathbf{k}_{e}}\left(\bar{v}_{e}\right)=\frac{1}{2} \cos ^{4} \theta \int d \tau \int d \tau^{\prime} \frac{G_{e f f}^{2}}{(2 \pi)^{6}} \frac{e^{-i \Delta M\left(\tau-\tau^{\prime}\right)}}{\omega_{v_{1}} \omega_{e}} e^{i\left(k_{v_{1}}+k_{e}\right)^{\lambda}\left[x(\tau)-x\left(\tau^{\prime}\right)\right]_{\lambda}} \\
& \times\left\{2\left[2 k_{e}^{(\alpha} k_{v_{1}}^{\beta)}-i \varepsilon^{\alpha \beta \gamma \rho}\left(k_{e}\right)_{\gamma}\left(k_{v_{1}}\right)_{\rho}\right] u_{\alpha}(\tau) u_{\beta}\left(\tau^{\prime}\right)-2\left(k_{e}\right)^{\lambda}\left(k_{v_{1}}\right)_{\lambda} u^{\delta}(\tau) u_{\delta}\left(\tau^{\prime}\right)\right\} \\
& +\frac{1}{2} \sin ^{4} \theta \int d \tau \int d \tau^{\prime} \frac{G_{e f f}^{2}}{(2 \pi)^{6}} \frac{e^{-i \Delta M\left(\tau-\tau^{\prime}\right)}}{\omega_{v_{2}} \omega_{e}} e^{i\left(k_{v_{2}}+k_{e}\right)^{\lambda}\left[x(\tau)-x\left(\tau^{\prime}\right)\right]_{\lambda}} \\
& \quad \times\left\{2\left[2 k_{e}^{(\alpha} k_{v_{2}}^{\beta)}-i \varepsilon^{\alpha \beta \gamma \rho}\left(k_{e}\right)_{\gamma}\left(k_{v_{2}}\right)_{\rho}\right] u_{\alpha}(\tau) u_{\beta}\left(\tau^{\prime}\right)-2\left(k_{e}\right)^{\lambda}\left(k_{v_{2}}\right) \lambda u^{\delta}(\tau) u_{\delta}\left(\tau^{\prime}\right)\right\} \\
& +\frac{1}{2} \cos ^{2} \theta \sin ^{2} \theta\left\{\int d \tau \int d \tau^{\prime} G_{e f f}^{2} e^{-i \Delta M\left(\tau-\tau^{\prime}\right)} u_{\alpha}(\tau) u_{\beta}\left(\tau^{\prime}\right)\right. \\
& \quad \times \sum_{\sigma_{e}, \sigma_{v}= \pm}\left[\bar{\psi}_{\mathbf{k}_{e}, \sigma_{e}}^{\left(+\omega_{e}\right)}(x(\tau)) \gamma^{\alpha}\left(1-\gamma^{5}\right) \psi_{-\mathbf{k}_{v},-\sigma_{v}}^{\left(-\omega_{v_{1}}\right)}(x(\tau)) \bar{\psi}_{-\mathbf{k}_{v},-\sigma_{v}}^{\left(-\omega_{v_{2}}\right)}\left(x\left(\tau^{\prime}\right)\right) \gamma^{\beta}\left(1-\gamma^{5}\right) \psi_{\mathbf{k}_{e}, \sigma_{e}}^{\left(+\omega_{e}\right)}\left(x\left(\tau^{\prime}\right)\right)\right] \\
& +c . c .\},
\end{aligned}
$$

where $\varepsilon^{\alpha \beta \gamma \delta}$ is the Levi-Civita pseudotensor (with $\varepsilon^{0123}=1$ ) and

$$
k_{e}^{(\alpha} k_{v_{j}}^{\beta)} \equiv\left(k_{e}^{\alpha} k_{v_{j}}^{\beta}+k_{e}^{\beta} k_{v_{j}}^{\alpha}\right) / 2 .
$$

Equation (2.18) will be the starting point of our next analysis.

\section{Calculation of the $\beta$-decay rate}

Let us exploit the notions and formalism of Sec. 2 to compute the $\beta$-decay of both static and uniformly accelerated neutrons. The two results shall then be compared in the limit of vanishing acceleration, showing that they are consistent with each other, as well as with the full-fledged field theoretical outcome of Ref. [12]. 


\subsection{Static neutron}

In the case of a static neutron, the worldline $x^{\alpha}(\tau)$ is simply given by

$$
x^{\alpha}(\tau)=\left(\tau, x^{1}, x^{2}, x^{3}\right),
$$

with $x^{i}=$ const. Accordingly, the four-velocity takes the form

$$
u^{\alpha}(\tau)=(1,0,0,0) .
$$

By plugging the above relations into the differential transition probability (2.18), after some tedious but straightforward calculations we get

$$
\begin{aligned}
& \frac{d \mathscr{P}^{n \rightarrow p}}{d^{3} \mathbf{k}_{v} d^{3} \mathbf{k}_{e}}\left(\bar{v}_{e}\right)=\cos ^{4} \theta\left(1+\frac{\mathbf{k}_{v} \cdot \mathbf{k}_{e}}{\omega_{v_{1}} \omega_{e}}\right) \int d \tau \int d \tau^{\prime} \frac{G_{e f f}^{2}}{(2 \pi)^{6}} e^{i\left(-\Delta M+\omega_{v_{1}}+\omega_{e}\right)\left(\tau-\tau^{\prime}\right)} \\
& +\sin ^{4} \theta\left(1+\frac{\mathbf{k}_{v} \cdot \mathbf{k}_{e}}{\omega_{v_{2}} \omega_{e}}\right) \int d \tau \int d \tau^{\prime} \frac{G_{e f f}^{2}}{(2 \pi)^{6}} e^{i\left(-\Delta M+\omega_{v_{2}}+\omega_{e}\right)\left(\tau-\tau^{\prime}\right)} \\
& +\frac{1}{2} \cos ^{2} \theta \sin ^{2} \theta\left\{\frac{\omega_{e}\left[\left|\mathbf{k}_{v}\right|^{2}+\left(\omega_{v_{1}}-m_{v_{1}}\right)\left(\omega_{v_{2}}-m_{v_{2}}\right)\right]+\mathbf{k}_{e} \cdot \mathbf{k}_{v}\left(\omega_{v_{1}}+\omega_{v_{2}}-m_{v_{1}}-m_{v_{2}}\right)}{\omega_{e} \sqrt{\omega_{v_{1}} \omega_{v_{2}}\left(\omega_{v_{1}}-m_{v_{1}}\right)\left(\omega_{v_{2}}-m_{v_{2}}\right)}}\right. \\
& \left.\quad \times \int d \tau \int d \tau^{\prime} \frac{G_{e f f}^{2}}{(2 \pi)^{6}} e^{i\left(-\Delta M+\omega_{e}\right)\left(\tau-\tau^{\prime}\right)} e^{i\left(\omega_{v_{1}} \tau-\omega_{v_{2}} \tau^{\prime}\right)}+c . c .\right\} .
\end{aligned}
$$

The above equation can be simplified by introducing the new coordinates

$$
\xi=\frac{\tau-\tau^{\prime}}{2}, \quad s=\frac{\tau+\tau^{\prime}}{2}
$$

in term of which the integrals in Eq. (3.3) can be decoupled, giving

$$
\begin{aligned}
& \frac{d \mathscr{P}^{n \rightarrow p}}{d^{3} \mathbf{k}_{v} d^{3} \mathbf{k}_{e}}\left(\bar{v}_{e}\right)=2 \cos ^{4} \theta\left(1+\frac{\mathbf{k}_{v} \cdot \mathbf{k}_{e}}{\omega_{v_{1}} \omega_{e}}\right) \int d s \int d \xi \frac{G_{e f f}^{2}}{(2 \pi)^{6}} e^{2 i\left(-\Delta M+\omega_{v_{1}}+\omega_{e}\right) \xi} \\
& +2 \sin ^{4} \theta\left(1+\frac{\mathbf{k}_{v} \cdot \mathbf{k}_{e}}{\omega_{v_{2}} \omega_{e}}\right) \int d s \int d \xi \frac{G_{e f f}^{2}}{(2 \pi)^{6}} e^{2 i\left(-\Delta M+\omega_{v_{2}}+\omega_{e}\right) \xi} \\
& +\cos ^{2} \theta \sin ^{2} \theta\left\{\frac{\omega_{e}\left[\left|\mathbf{k}_{v}\right|^{2}+\left(\omega_{v_{1}}-m_{v_{1}}\right)\left(\omega_{v_{2}}-m_{v_{2}}\right)\right]+\mathbf{k}_{e} \cdot \mathbf{k}_{v}\left(\omega_{v_{1}}+\omega_{v_{2}}-m_{v_{1}}-m_{v_{2}}\right)}{\omega_{e} \sqrt{\omega_{v_{1}} \omega_{v_{2}}\left(\omega_{v_{1}}-m_{v_{1}}\right)\left(\omega_{v_{2}}-m_{v_{2}}\right)}}\right. \\
& \left.\times \int d s e^{i\left(\omega_{v_{1}}-\omega_{v_{2}}\right) s} \int d \xi \frac{G_{e f f}^{2}}{(2 \pi)^{6}} e^{i\left(-2 \Delta M+\omega_{v_{1}}+\omega_{v_{2}}+2 \omega_{e}\right) \xi}+\text { c.c. }\right\} .
\end{aligned}
$$

Note that, for $\theta \rightarrow 0$ and/or $m_{v_{1}} \rightarrow m_{v_{2}}$, the result of Ref. [10] is straightforwardly recovered, as expected in the absence of mixing ${ }^{5}$.

${ }^{5}$ More precisely, since we are computing the averaged $\beta$-decay rate, the result (3.7) is one half of the outcome of Ref. [10] for $\theta \rightarrow 0$. 
Let us emphasize that the presence of the off-diagonal term in Eqs. (3.3) and (3.5) is a direct consequence of describing the outgoing antineutrino via the flavor eigenstate $\left|\bar{v}_{e}\right\rangle$. On the other hand, by using mass eigenstates, one would simply obtain the inchoerent sum of the probabilities for each massive antineutrino $\bar{v}_{i}$ multiplied by the square of the associate mixing matrix element $U_{e i}$, i.e.

$$
\begin{aligned}
\frac{d \mathscr{P}^{n \rightarrow p}}{d^{3} \widetilde{\mathbf{k}}_{v} d^{3} \widetilde{\mathbf{k}}_{e}} \equiv & \frac{d \mathscr{P}^{n \rightarrow p}}{d^{3} \widetilde{\mathbf{k}}_{v} d^{3} \widetilde{\mathbf{k}}_{e}}\left(\bar{v}_{1}\right)+\frac{d \mathscr{P}^{n \rightarrow p}}{d^{3} \widetilde{\mathbf{k}}_{v} d^{3} \widetilde{\mathbf{k}}_{e}}\left(\bar{v}_{2}\right) \\
= & 2 \cos ^{2} \theta\left(1+\frac{\mathbf{k}_{v} \cdot \mathbf{k}_{e}}{\omega_{v_{1}} \omega_{e}}\right) \int d s \int d \xi \frac{G_{e f f}^{2}}{(2 \pi)^{6}} e^{2 i\left(-\Delta M+\omega_{v_{1}}+\omega_{e}\right) \xi} \\
& +2 \sin ^{2} \theta\left(1+\frac{\mathbf{k}_{v} \cdot \mathbf{k}_{e}}{\omega_{v_{2}} \omega_{e}}\right) \int d s \int d \xi \frac{G_{e f f}^{2}}{(2 \pi)^{6}} e^{2 i\left(-\Delta M+\omega_{v_{2}}+\omega_{e}\right) \xi}
\end{aligned}
$$

Clearly, this latter setting fails to account for the quantum interference between the two mass states. A more detailed discussion on this can be found in Refs. [2, 5, 6, 7].

In order to compare our result with Ref. [12], let us now perform the $\xi$ - and $s$ - integrals over infinite time intervals. Denoting by $T=2 \pi \delta(0)$ the (infinite) proper time of the nucleon, Eq. (3.5) becomes

$$
\begin{aligned}
\frac{d \Gamma^{n \rightarrow p}}{d^{3} \mathbf{k}_{v} d^{3} \mathbf{k}_{e}}\left(\bar{v}_{e}\right)= & \cos ^{4} \theta \frac{G_{e f f}^{2}}{(2 \pi)^{5}}\left(1+\frac{\mathbf{k}_{v} \cdot \mathbf{k}_{e}}{\omega_{v_{1}} \omega_{e}}\right) \delta\left(\omega_{v_{1}}+\omega_{e}-\Delta M\right) \\
& +\sin ^{4} \theta \frac{G_{e f f}^{2}}{(2 \pi)^{5}}\left(1+\frac{\mathbf{k}_{v} \cdot \mathbf{k}_{e}}{\omega_{v_{2}} \omega_{e}}\right) \delta\left(\omega_{v_{2}}+\omega_{e}-\Delta M\right),
\end{aligned}
$$

where

$$
\Gamma^{n \rightarrow p}=\mathscr{P}^{n \rightarrow p} / T
$$

is the transition probability per proper time. Note that in this case the off-diagonal contribution vanishes, as it is equal to a Dirac delta of non-null argument $\left(\omega_{v_{1}} \neq \omega_{v_{2}}\right)$ resulting from the $s$ integration (see Sec. 3.2 for a comparison with the case of an accelerated neutron). Equivalently, in terms of the old coordinates $\tau, \tau^{\prime}$, it is easy to see that this term consists of the product of two Dirac deltas picked up around the two different energy values $\omega_{v_{1}}+\omega_{e}-\Delta M$ and $\omega_{v_{2}}+\omega_{e}-\Delta M$, respectively.

The obtained formula (3.7) allows us to point out an interesting consideration about the stability of static (or, more generally, inertial) protons. Starting from the outlined picture, indeed, we can analyze the decay of a proton into a neutron by simply reversing the rôles of the two particles. In that case, the mass difference $\Delta M$ would acquire a negative sign, resulting in strictly positive arguments of the two Dirac deltas in Eq. (3.7), and, thus, in a vanishing expression for the decay rate, in agreement with Standard Model.

Now, by choosing a spherical coordinate system with respect to $\mathbf{k}_{v}$ and integrating over the electron and antineutrino momenta, the terms containing the product $\mathbf{k}_{v} \cdot \mathbf{k}_{e}$ in Eq. (3.7) vanish, 
yielding

$$
\begin{aligned}
d \Gamma^{n \rightarrow p}\left(\bar{v}_{e}\right)= & \cos ^{4} \theta \frac{G_{e f f}^{2}}{2 \pi^{3}}\left|\mathbf{k}_{v}\right|^{2}\left|\mathbf{k}_{e}\right|^{2} \delta\left(\omega_{v_{1}}+\omega_{e}-\Delta M\right) d k_{v} d k_{e} \\
& +\sin ^{4} \theta \frac{G_{e f f}^{2}}{2 \pi^{3}}\left|\mathbf{k}_{v}\right|^{2}\left|\mathbf{k}_{e}\right|^{2} \delta\left(\omega_{v_{2}}+\omega_{e}-\Delta M\right) d k_{v} d k_{e} .
\end{aligned}
$$

The spectrum for the $\beta$-decay process is then

$$
\begin{aligned}
\frac{d \Gamma^{n \rightarrow p}}{d \omega_{e}}\left(\bar{v}_{e}\right)= & \cos ^{4} \theta \frac{G_{e f f}^{2}}{2 \pi^{3}} \sqrt{\left(\Delta M-\omega_{e}\right)^{2}-m_{v_{1}}^{2}}\left|\mathbf{k}_{e}\right| \omega_{e}\left(\Delta M-\omega_{e}\right) \\
& +\sin ^{4} \theta \frac{G_{e f f}^{2}}{2 \pi^{3}} \sqrt{\left(\Delta M-\omega_{e}\right)^{2}-m_{v_{2}}^{2}}\left|\mathbf{k}_{e}\right| \omega_{e}\left(\Delta M-\omega_{e}\right) .
\end{aligned}
$$

To the linear order in $m_{v_{j}} / \omega_{v_{j}}$, the above relation takes the form

$$
\frac{d \Gamma^{n \rightarrow p}}{d \omega_{e}}\left(\bar{v}_{e}\right)=\left(1-\frac{1}{2} \sin ^{2} 2 \theta\right) \frac{G_{e f f}^{2}}{2 \pi^{3}}\left|\mathbf{k}_{e}\right| \omega_{e}\left(\Delta M-\omega_{e}\right)^{2}+\mathscr{O}\left(\frac{m_{v}}{\omega_{v}}\right)^{2},
$$

which is simply the product of the spectrum for massless antineutrino, multiplied by the average survival probability. This could be somehow expected, since when we perform the time integration and take the asymptotic limit $T \rightarrow \infty$, we are effectively averaging the oscillation probability of the antineutrino.

It is now worth noting that Eq. (3.11) is consistent with the result obtained in the relativistic limit in Ref. [12] by using the generalized coherent flavor states (instead of Pontecorvo states) for mixed antineutrinos and a weak Lagrangian which properly takes into account the different quark compositions of the neutron and proton. Specifically, we have to compare Eq. (3.11) with Eq. (4.41) of Ref. [12], here recast in our notation

$$
\frac{d \Gamma^{n \rightarrow p}}{d \omega_{e}}\left(\bar{v}_{e}\right)=\left(1-\frac{1}{2} \sin ^{2} 2 \theta\right) \frac{G_{F}^{2} V_{u d}^{2}}{2 \pi^{3}}\left(f^{2}+3 g^{2}\right)\left|\mathbf{k}_{e}\right| \omega_{e}\left(\Delta M-\omega_{e}\right)^{2}+\mathscr{O}\left(\frac{m_{v}}{\omega_{v}}\right)^{2},
$$

where $G_{F} \simeq G_{e f f} / 2$ is the Fermi coupling constant ${ }^{6}, V_{u d} \simeq 1$ is the element of the CKM matrix describing the transition amplitude from a quark down to a quark up, and $f \simeq 1, g \gtrsim 1$ are the form factors [13].

So far, we have only considered the decay channel (1.1): due to the asymptotic occurrence of flavor oscillations, however, the process $n \rightarrow p+e^{-}+\bar{v}_{\mu}$ has a non-vanishing probability as well. By following similar calculations, one can show that the energy spectrum for this process is

$$
\frac{d \Gamma^{n \rightarrow p}}{d \omega_{e}}\left(\bar{v}_{\mu}\right)=\frac{1}{2} \sin ^{2} 2 \theta \frac{G_{e f f}^{2}}{2 \pi^{3}}\left|\mathbf{k}_{e}\right| \omega_{e}\left(\Delta M-\omega_{e}\right)^{2}+\mathscr{O}\left(\frac{m_{v}}{\omega_{v}}\right)^{2},
$$

\footnotetext{
${ }^{6}$ The relation between the effective coupling constant $G_{\text {eff }}$ and the Fermi coupling constant $G_{F}$ can be found by imposing that the neutron proper lifetime is $887 \mathrm{~s}$, i.e. $\Gamma^{n \rightarrow p} \simeq 1 / 887 \mathrm{~s}^{-1}$.
} 
which is the the spectrum for massless antineutrino times the average oscillation probability. Then, the total neutron $\beta$-decay spectrum is simply given by the sum over the two flavors, i.e.

$$
\begin{aligned}
\frac{d \Gamma^{n \rightarrow p}}{d \omega_{e}} & \equiv \frac{d \Gamma^{n \rightarrow p}}{d \omega_{e}}\left(\bar{v}_{e}\right)+\frac{d \Gamma^{n \rightarrow p}}{d \omega_{e}}\left(\bar{v}_{\mu}\right) \\
& =\frac{G_{e f f}^{2}}{2 \pi^{3}}\left|\mathbf{k}_{e}\right| \omega_{e}\left(\Delta M-\omega_{e}\right)^{2}+\mathscr{O}\left(\frac{m_{v}}{\omega_{v}}\right)^{2}
\end{aligned}
$$

which matches the prediction of the (minimal) Standard Model with massless neutrino.

Finally, let us note that, even though $d \Gamma^{n \rightarrow p}\left(\bar{v}_{e}\right) / d \omega_{e} \neq d \Gamma^{n \rightarrow p}\left(\bar{v}_{1}\right) / d \omega_{e}+d \Gamma^{n \rightarrow p}\left(\bar{v}_{2}\right) / d \omega_{e}$, the following equality holds:

$$
\frac{d \Gamma^{n \rightarrow p}}{d \omega_{e}}\left(\bar{v}_{e}\right)+\frac{d \Gamma^{n \rightarrow p}}{d \omega_{e}}\left(\bar{v}_{e}\right)=\frac{d \Gamma^{n \rightarrow p}}{d \omega_{e}}\left(\bar{v}_{1}\right)+\frac{d \Gamma^{n \rightarrow p}}{d \omega_{e}}\left(\bar{v}_{2}\right),
$$

regardless of the approximation of relativistic neutrinos. As explained in Ref. [7], this is related to the conservation of the total lepton number both in the presence and in the absence of flavor mixing.

\subsection{Accelerated neutron}

Let us now analyze how the transition probability (3.5) gets modified for an accelerated neutron [14]. The computation of the energy spectrum requires further analysis and will be presented elsewhere [15].

Denoting by $a$ the proper acceleration of the nucleon and assuming the motion to be along the $z$-axis, the worldline in the usual Minkowski coordinates reads [10]

$$
x^{\alpha}(\tau)=\left(a^{-1} \sinh a \tau, 0,0, a^{-1} \cosh a \tau\right)
$$

the corresponding four-velocity of which is

$$
u^{\alpha}(\tau)=(\cosh a \tau, 0,0, \sinh a \tau) .
$$

As in Sec. 3.1, it comes in handy to introduce the coordinates $\xi$ and $s$, in terms of which one has

$$
\begin{gathered}
{\left[x(\tau)-x\left(\tau^{\prime}\right)\right]^{\lambda}=2 a^{-1} \sinh (a \xi)[\cosh (a s), 0,0, \sinh (a s)]=2 a^{-1} \sinh (a \xi) u^{\lambda}(s)} \\
u^{\alpha}(\tau)=\cosh (a \xi) u^{\alpha}(s)+a \sinh (a \xi) x^{\alpha}(s), \quad u^{\alpha}\left(\tau^{\prime}\right)=\cosh (a \xi) u^{\alpha}(s)-a \sinh (a \xi) x^{\alpha}(s), \\
u^{\alpha}(\tau) u_{\alpha}\left(\tau^{\prime}\right)=\cosh (2 a \xi) .
\end{gathered}
$$

However, in this case the decoupling of the $\xi$ - and $s$-integrals requires a further step, namely a boost in the $z$-direction to the inertial frame instantaneously at rest with the accelerated nucleon current at the proper time $s$. In so doing, the four-momenta of the emitted fermions take the form

$$
k^{\alpha} \rightarrow \widetilde{k}^{\alpha} \equiv(\widetilde{\boldsymbol{\omega}}, \widetilde{\mathbf{k}})=\left[k^{\lambda} u_{\lambda}(s), k^{x}, k^{y},-a k^{\lambda} x_{\lambda}(s)\right] .
$$

Note that, for $a \rightarrow 0$, we have $\widetilde{k}^{\alpha} \rightarrow k^{\alpha}$, as it should be. 
Now, by plugging Eqs. (3.18) - (3.21) into (2.18), we obtain

$$
\begin{aligned}
& \frac{d \mathscr{P}^{n \rightarrow p}}{d^{3} \mathbf{k}_{v} d^{3} \mathbf{k}_{e}}\left(\bar{v}_{e}\right)=\frac{2}{\omega_{v_{1}} \omega_{e}} \cos ^{4} \theta \int d s \int d \xi \frac{G_{e f f}^{2}}{(2 \pi)^{6}} e^{2 i\left[-\Delta M \xi+a^{-1} \sinh (a \xi)\left(\widetilde{\omega}_{v_{1}}+\widetilde{\omega}_{e}\right)\right]} \\
& \times\left[\widetilde{\omega}_{v_{1}} \widetilde{\omega}_{e}+\widetilde{k}_{v}^{z} \widetilde{k}_{e}^{z}+\cosh (2 a \xi) \widetilde{\mathbf{k}}_{v}^{\perp} \cdot \widetilde{\mathbf{k}}_{e}^{\perp}+i \sinh (2 a \xi)\left(\widetilde{\mathbf{k}}_{v} \wedge \widetilde{\mathbf{k}}_{e}\right)^{z}\right] \\
& +\frac{2}{\omega_{v_{2}} \omega_{e}} \sin ^{4} \theta \int d s \int d \xi \frac{G_{e f f}^{2}}{(2 \pi)^{6}} e^{2 i\left[-\Delta M \xi+a^{-1} \sinh (a \xi)\left(\widetilde{\omega}_{v_{2}}+\widetilde{\omega}_{e}\right)\right]} \\
& \times\left[\widetilde{\omega}_{v_{2}} \widetilde{\omega}_{e}+\widetilde{k}_{v}^{z} \widetilde{k}_{e}^{z}+\cosh (2 a \xi) \widetilde{\mathbf{k}}_{v}^{\perp} \cdot \widetilde{\mathbf{k}}_{e}^{\perp}+i \sinh (2 a \xi)\left(\widetilde{\mathbf{k}}_{v} \wedge \widetilde{\mathbf{k}}_{e}\right)^{z}\right] \\
& +\cos ^{2} \theta \sin ^{2} \theta \frac{1}{\omega_{e} \sqrt{\omega_{v_{1}} \omega_{v_{2}}\left(\omega_{v_{1}}-m_{v_{1}}\right)\left(\omega_{v_{2}}-m_{v_{2}}\right)}} \int d s \int d \xi \frac{G_{e f f}^{2}}{(2 \pi)^{6}}\left\{e^{-2 i \Delta M \xi}\right. \\
& \times e^{2 i a^{-1} \sinh (a \xi) \widetilde{\omega}_{e}} e^{i a^{-1}\left\{\sinh [a(s+\xi)] \omega_{v_{1}}-\sinh [a(s-\xi)] \omega_{v_{2}}\right\}} e^{-2 i a^{-1} \sinh (a \xi) \sinh (a s) k_{v}^{z}} \\
& \times\left\{\cosh (2 a s)\left[\omega_{e}\left(\left|\mathbf{k}_{v}\right|^{2}+\left(\omega_{v_{1}}-m_{v_{1}}\right)\left(\omega_{v_{2}}-m_{v_{2}}\right)\right)+k_{v}^{z} k_{e}^{z}\left(\omega_{v_{1}}+\omega_{v_{2}}-m_{v_{2}}-m_{v_{2}}\right)\right]\right. \\
& +\left(\omega_{v_{1}}+\omega_{v_{2}}-m_{v_{2}}-m_{v_{2}}\right)\left[\cosh (2 a \xi) \mathbf{k}_{v}^{\perp} \cdot \mathbf{k}_{e}^{\perp}+i \sinh (2 a \xi)\left(\mathbf{k}_{v} \wedge \mathbf{k}_{e}\right)^{z}\right] \\
& \left.\left.-\sinh (2 a s)\left[k_{e}^{z}\left(\left|\mathbf{k}_{v}\right|^{2}+\left(\omega_{v_{1}}-m_{v_{1}}\right)\left(\omega_{v_{2}}-m_{v_{2}}\right)\right)+k_{v}^{z} \omega_{e}\left(\omega_{v_{1}}+\omega_{v_{2}}-m_{v_{1}}-m_{v_{2}}\right)\right]\right\}+c . c .\right\},
\end{aligned}
$$

where $\mathbf{k}^{\perp} \equiv\left(k^{x}, k^{y}\right)$ is the transverse momentum and $\wedge$ denotes the (three) vector product. Furthermore, we have exploited the invariance of the product $\widetilde{k}_{\lambda} \widetilde{k}^{\lambda}$ under the boost (3.21). Clearly, where left untouched, the frequencies $\omega_{e\left(v_{j}\right)}$ and the momenta $\mathbf{k}_{e(v)}$ must be regarded as functions of the new variables $\widetilde{k}_{e\left(v_{j}\right)}^{\alpha}$ through the transformation (3.21), from which one obtains

$$
\begin{aligned}
d^{3} \widetilde{\mathbf{k}}_{v} d^{3} \widetilde{\mathbf{k}}_{e} & =d^{3} \mathbf{k}_{v} d^{3} \mathbf{k}_{e} \frac{\left[\omega_{e} \cosh (a s)-k_{e}^{z} \sinh (a s)\right]\left[\omega_{v} \cosh (a s)-k_{v}^{z} \sinh (a s)\right]}{\omega_{v} \omega_{e}} \\
& =d^{3} \mathbf{k}_{v} d^{3} \mathbf{k}_{e} \frac{\widetilde{\omega}_{v} \widetilde{\omega}_{e}}{\omega_{v} \omega_{e}} .
\end{aligned}
$$

For infinite nucleon proper time, the evaluation of the integrals in the two diagonal terms in Eq. (3.22) is quite straightforward, as shown in Ref. [10]. By contrast, the simultaneous dependence on $m_{v_{1}}$ and $m_{v_{2}}$ makes the interference contribution tougher to treat, since the $\xi$ - and $s$-integrals cannot be decoupled just as easily (a first estimate was exhibited in Ref. [2] for the case of the inverse $\beta$-decay of an accelerated proton). Preliminary calculations [15] seem to indicate that, due to the acceleration, the two Dirac deltas arising from the $\tau$ - and $\tau^{\prime}$-integrations in the last term of Eq. (3.5) would smear out over the energy scale (see the discussion after Eq. (3.7)). The ensuing overlapping would thus be responsible for a non-trivial interference between the two mass eigenstates (contrary to what happens in Eq. (3.5)), despite being $\omega_{v_{1}} \neq \omega_{v_{2}}$.

In order to check the consistency of our formalism, let us now compute the $a \rightarrow 0$ limit of 
Eq. (3.22). By explicit calculations, we obtain

$$
\begin{aligned}
& \frac{d \mathscr{P}^{n \rightarrow p}}{d^{3} \mathbf{k}_{v} d^{3} \mathbf{k}_{e}}\left(\bar{v}_{e}\right)=\frac{2}{\omega_{v_{1}} \omega_{e}} \cos ^{4} \theta \int d s \int d \xi \frac{G_{e f f}^{2}}{(2 \pi)^{6}} e^{2 i\left(-\Delta M+\omega_{v_{1}}+\omega_{e}\right) \xi}\left(\omega_{v_{1}} \omega_{e}+k_{v}^{z} k_{e}^{z}+\mathbf{k}_{v}^{\perp} \cdot \mathbf{k}_{e}^{\perp}\right) \\
& \quad+\frac{2}{\omega_{v_{2}} \omega_{e}} \cos ^{4} \theta \int d s \int d \xi \frac{G_{e f f}^{2}}{(2 \pi)^{6}} e^{2 i\left(-\Delta M+\omega_{v_{2}}+\omega_{e}\right) \xi}\left(\omega_{v_{2}} \omega_{e}+k_{v}^{z} k_{e}^{z}+\mathbf{k}_{v}^{\perp} \cdot \mathbf{k}_{e}^{\perp}\right) \\
& \quad+\cos ^{2} \theta \sin ^{2} \theta \frac{1}{\omega_{e} \sqrt{\omega_{v_{1}} \omega_{v_{2}}\left(\omega_{v_{1}}-m_{v_{1}}\right)\left(\omega_{v_{2}}-m_{v_{2}}\right)}} \int d s \int d \xi \frac{G_{e f f}^{2}}{(2 \pi)^{6}}\left\{e^{-2 i \Delta M \xi}\right. \\
& \quad \times e^{2 i \omega_{e} \xi} e^{i\left[\omega_{v_{1}}(s+\xi)-\omega_{v_{2}}(s-\xi)\right]}\left\{\omega_{e}\left[\left|\mathbf{k}_{v}\right|^{2}+\left(\omega_{v_{1}}-m_{v_{1}}\right)\left(\omega_{v_{2}}-m_{v_{2}}\right)\right]\right. \\
& \left.\left.+k_{v}^{z} k_{e}^{z}\left(\omega_{v_{1}}+\omega_{v_{2}}-m_{v_{2}}-m_{v_{2}}\right)+\left(\mathbf{k}_{v}^{\perp} \cdot \mathbf{k}_{e}^{\perp}\right)\left(\omega_{v_{1}}+\omega_{v_{2}}-m_{v_{2}}-m_{v_{2}}\right)\right\}+c . c .\right\}
\end{aligned}
$$

which exactly reproduces the result in Eq. (3.5).

A comment is in order at this stage: as a first step of our analysis, by considering the decay of a static neutron, we have found that our formalism is consistent with Standard Model expectations and with a natural description of neutrino flavor oscillations, provided one adopts flavor states as fundamental representation for antineutrinos. Next, we have addressed the case of an accelerated neutron, showing that the previous framework is correctly recovered (both at physical and mathematical levels) in the limit of vanishing acceleration. In light of this, we can look at the following scenario. Consider a static neutron: since it decays through the weak interaction, the conservation (at tree level) of the family lepton number ensures that the antineutrino will be emitted with definite flavor. Let us now accelerate the neutron: what does our formalism predict for the antineutrino? Will it still appear with definite flavor? Will it be produced as a massive antineutrino? Since the formalism can accommodate arbitrarily small values of the acceleration, we can focus on a barely accelerated particle: in this context, we have no a priori reason to believe that a slight change in the kinematics of the neutron may affect the properties of the decay products so drastically as to alter the nature of the antineutrino. Actually, these properties $d o$ depend on the type of interaction - which remains unchanged - rather than the motion of the parent particle. Thus, the answer is that the outgoing antineutrino will still appear in a definite flavor eigenstate.

Let us now observe that all the above considerations also apply to the analysis of Refs. [2, 6, 7], where the same formalism is employed for studying the inverse $\beta$-decay of accelerated protons. Hence, the adoption of flavor eigenstates for describing asymptotic neutrinos is physically wellgrounded and correct even in that framework, contrary to what stated elsewhere in the literature.

\section{Conclusions and Discussion}

Since Pontecorvo's pioneering work [9], the theoretical foundations of neutrino mixing have been studied with great care. Phenomenological and experimental developments have successfully confirmed the original idea of the occurrence of the phenomena of flavor mixing and oscillations, thus opening new scenarios beyond the Standard Model. Challenging problems, however, remain unsolved. Among these, the question of whether to consider mass or flavor states as fundamental 
representation for asymptotic neutrinos is still a vibrant subject of investigation $[2,6,7,11,12,16$, 17, 18]. Along this line, in the present work we have attempted to answer this question by studying the rôle of neutrino mixing in weak decay processes. As a test bench, we have considered the $\beta$-decay of both static and accelerated neutrons. In the first case, we have found that the adoption of flavor states leads to expressions for the decay rate and energy spectrum which agree with the minimal Standard Model predictions in the relativistic limit, as well as with a natural description of flavor oscillations. Then, we have extended our analysis to non-inertial neutrons, showing the formalism to be well-defined and consistent with the above findings for vanishing acceleration Beyond its intrinsic interest, we have discussed the relevance of our result in connection with the recent controversy on the very nature of asymptotic neutrinos in the context of the inverse $\beta$-decay of accelerated protons [1, 2, 4]. Clearly, several aspects remain to be addressed, including a fullfledged QFT treatment of the problem which accounts for the description of the accelerating source and for acceleration-induced effects on neutrino mixing and oscillations [19, 20].

Apart from theoretical investigation, let us remark that important pieces of information on the dichotomy between flavor and mass neutrino states are expected to be provided by experiments. In Ref. [12] and therein, indeed, it has been been shown that the spectrum of the Tritium $\beta$-decay near the end-point energy is highly sensitive to whether neutrinos are flavor or mass eigenstates. Similar considerations apply to the neutrino capture by Tritium as well. Therefore, data from recent neutrino experiments such as KATRIN (which is projected to set an upper limit to the electron antineutrino mass by examining the spectrum of electrons emitted from the Tritium decay) and PTOLEMY (which aims to detect the relic neutrino background through a combination of a large area surface-deposition tritium target) may help to make substantial progress towards resolving the controversy.

Finally, we emphasize that the present formalism might be of some relevance for a deeper understanding of how gravity influences decay processes and neutrino oscillations [21, 22, 23, $24,25,26,27]$. Although a more rigorous curved-spacetime calculation is required to address these situations, the present study may provide a good approximation in the case of moderate gravitational field, for which one can naively treat the decaying particle as being accelerated in flat spacetime. This and other aspects will be investigated in more detail in future works.

\section{References}

[1] D. V. Ahluwalia, L. Labun and G. Torrieri, Neutrino mixing in accelerated proton decays, Eur. Phys. J. A 52, 189 (2016).

[2] M. Blasone, G. Lambiase, G. G. Luciano and L. Petruzziello, Role of neutrino mixing in accelerated proton decay, Phys. Rev. D 97, 105008 (2018).

[3] M. Blasone, G. Lambiase, G. G. Luciano and L. Petruzziello, Neutrino mixing and general covariance in the inverse $\beta$ decay, PoS CORFU 2017, 198 (2018).

[4] G. Cozzella, S. A. Fulling, A. G. S. Landulfo, G. E. A. Matsas and D. A. T. Vanzella, Unruh effect for mixing neutrinos, Phys. Rev. D 97, 105022 (2018).

[5] M. Blasone, G. Lambiase, G. G. Luciano and L. Petruzziello, Remarks on the Unruh effect with mixed neutrinos, J. Phys. Conf. Ser. 1275 , 012063 (2019). 
[6] M. Blasone, G. Lambiase, G. G. Luciano and L. Petruzziello, Neutrino oscillations in the Unruh radiation, Phys. Lett. B 800, 135083 (2020).

[7] M. Blasone, G. Lambiase, G. G. Luciano and L. Petruzziello, On the beta-decay of the accelerated proton and neutrino oscillations: a three-flavor description with CP violation, Eur. Phys. J. C 80, 130 (2020).

[8] W. G. Unruh, Notes on black hole evaporation, Phys. Rev. D 14, 870 (1976).

[9] B. Pontecorvo, Neutrino Experiments and the Problem of Conservation of Leptonic Charge, Sov. Phys. JETP 26, 984 (1968) [Zh. Eksp. Teor. Fiz. 53, 1717 (1967)].

[10] D. A. T. Vanzella and G. E. A. Matsas, Weak decay of uniformly accelerated protons and related processes, Phys. Rev. D 63, 014010 (2001).

[11] M. Blasone and G. Vitiello, Quantum field theory of fermion mixing, Annals Phys. 244, 283 (1995).

[12] C. Y. Lee, Neutrinos as flavor eigenstates: Interactions and oscillations in neutron beta decay, arXiv:1709.06306 [hep-ph].

[13] M. Tanabashi et al. [Particle Data Group], Review of Particle Physics, Phys. Rev. D 98, 030001 (2018).

[14] J. Anandan and C. R. Hagen, Neutron acceleration in uniform electromagnetic fields, Phys. Rev. A 50, 2860 (1994).

[15] G. G. Luciano, in preparation.

[16] C. Giunti and C. W. Kim, Fundamentals of Neutrino Physics and Astrophysics, Oxford University Press, Oxford (2007).

[17] M. Blasone, G. G. Luciano, L. Petruzziello and L. Smaldone, Casimir effect for mixed fields, Phys. Lett. B 786, 278 (2018).

[18] M. Blasone, L. Smaldone and G. Vitiello, Flavor neutrino states for pedestrians, J. Phys. Conf. Ser. 1275, 012023 (2019).

[19] M. Blasone, G. Lambiase and G. G. Luciano, Nonthermal signature of the Unruh effect in field mixing, Phys. Rev. D 96, 025023 (2017); Non-thermal Unruh radiation for flavour neutrinos, J. Phys. Conf. Ser. 956, 012021 (2018).

[20] M. Blasone, G. Lambiase, G. G. Luciano and L. Petruzziello, Neutrino oscillations in accelerated frames, EPL 124, 51001 (2018).

[21] D. V. Ahluwalia and C. Burgard, Gravitationally induced quantum mechanical phases and neutrino oscillations in astrophysical environments, Gen. Rel. Grav. 28, 1161 (1996).

[22] C. Y. Cardall and G. M. Fuller, Neutrino oscillations in curved space-time: An Heuristic treatment, Phys. Rev. D 55, 7960 (1997).

[23] G. Lambiase, Space-time fluctuations induced by D-branes and their effects on neutrino oscillations, Gen. Rel. Grav. 34, 1437 (2002).

[24] G. Lambiase, G. Papini, R. Punzi and G. Scarpetta, Neutrino optics and oscillations in gravitational fields, Phys. Rev. D 71, 073011 (2005).

[25] G. Barenboim and N. E. Mavromatos, Decoherent neutrino mixing, dark energy and matter-antimatter asymmetry, Phys. Rev. D 70, 093015 (2004). 
[26] J. Alexandre, K. Farakos, N. E. Mavromatos and P. Pasipoularides, Neutrino oscillations in a stochastic model for space-time foam, Phys. Rev. D 77, 105001 (2008).

[27] L. Buoninfante, G. G. Luciano, L. Petruzziello and L. Smaldone, Neutrino oscillations in extended theories of gravity, Phys. Rev. D 101, 024016 (2020). 